\title{
Characterization of Each St and Y Genome Chromosome of Roegneria grandis Based on Newly Developed FISH Markers
}

 \\ Yi Wang $^{\mathrm{a}}$ Houyang Kang ${ }^{\mathrm{a}}$ Jiale $\mathrm{Lu}^{\mathrm{a}}$ Yonghong Zhou ${ }^{\mathrm{a}, \mathrm{b}}$

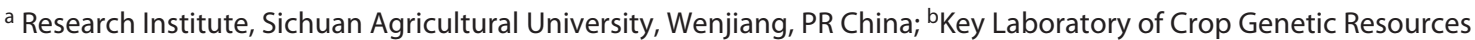 \\ and Improvement, Ministry of Education, Sichuan Agricultural University, Wenjiang, PR China; ' Horticulture \\ Research Institute, Sichuan Academy of Agricultural Sciences, Jinjiang, PR China
}

\section{Keywords \\ Comparative cytogenetics - FISH-based karyotype · \\ Genome structure variation · Nonhomologous \\ chromosomes $\cdot$ Repetitive sequence}

\begin{abstract}
The genera of the tribe Triticeae (family Poaceae), constituting many economically important plants with abundant genetic resources, carry genomes such as $\mathrm{St}, \mathrm{H}, \mathrm{P}$, and $\mathrm{Y}$. The genome symbol of Roegneria C. Koch (Triticeae) is StY. The St and $Y$ genomes are crucial in Triticeae, and tetraploid StY species participate extensively in polyploid speciation. Characterization of $\mathrm{St}$ and $\mathrm{Y}$ nonhomologous chromosomes in StY-genome species could help understand variation in the chromosome structure and differentiation of StY-containing species. However, the high genetic affinity between St and $\mathrm{Y}$ genome and the deficiency of a complete set of StY nonhomologous probes limit the identification of St and $\mathrm{Y}$ genomes and variation of chromosome structures among Roegneria species. We aimed to identify St- and Y-enhanced repeat clusters and to study whether homoeologous chromosomes between $S t$ and $Y$ genomes could be accurately identified due to high affinity. We employed comparative
\end{abstract}

karger@karger.com

(c) 2021 S. Karger AG, Basel

www.karger.com $/ \mathrm{cgr}$

Karger" genome analyses to identify St- and Y-enhanced repeat clusters and generated a FISH-based karyotype of $R$. grandis (Keng), one of the taxonomically controversial StY species, for the first time. We explored 4 novel repeat clusters (StY_34, StY_107, StY_90, and StY_93), which could specifically identify individual St and $Y$ nonhomologous chromosomes. The clusters StY_107 and StY_90 could identify St and Y addition/ substitution chromosomes against common wheat genetic backgrounds. The chromosomes V_St, VII_St, I_Y, V_Y, and VII_Y displayed similar probe distribution patterns in the proximal region, indicating that the high affinity between $\mathrm{St}$ and $Y$ genome might result from chromosome rearrangements or transposable element insertion among $\mathrm{V} \_\mathrm{St} / \mathrm{Y}, \mathrm{VII}$ $\mathrm{St} / \mathrm{Y}$, and I_Y chromosomes during allopolyploidization. Our results can be used to employ FISH further to uncover the precise karyotype based on colinearity of Triticeae species by using the wheat karyotype as reference, to analyze diverse populations of the same species to understand the intraspecific structural changes, and to generate the karyotype of different StY-containing species to understand the interspecific chromosome variation.

(c) 2021 S. Karger AG, Basel

Dandan Wu and Xiaoxia Zhu contributed equally to this work.
Correspondence to:

Yonghong Zhou, zhouyh@ sicau.edu.cn 


\section{Introduction}

The tribe Triticeae (family Poaceae) includes economic crop species (e.g., wheat, rye, triticale, and barley) and forage species (e.g., Roegneria C. Koch, Agropyron Gaertn., Leymus Hochst) with abundant genetic resources. The fundamental genomic constitutions in Triticeae include St, H, P, and Y genomes, which are derived from Pseudoroegneria (Nevski) Löve, Hordeum L., Agropyron, and an unknown diploid donor, respectively [Jensen, 1990; Torabinejad and Mueller, 1993; Wang et al., 1994; Wang and $\mathrm{Lu}, 2014]$. The genome symbol of the Roegneria genus is StY [Yen and Yang, 2011]. The polyphyletic allotetraploid $\mathrm{StY}$ species served as the maternal donor of hexaploid genera like Campeistachys Drobov (StStYYHH), Kengyilia C.Yen \& J.L.Yang (StStYYPP), and Anthosachne Steudel (StStYYWW). Further studies characterizing the parental St and Y genomes in StY species will help elucidate the classification and variation of StY-containing species.

The taxonomic status of Roegneria species is controversial for the following reasons: (1) similar plant morphological characteristics are present in St-containing genera Roegneria, Elymus L., and Campeiostachys such as spikelet number per node, lemma, and other features [Yen and Yang, 2011, 2013], (2) the polyphyletic origin of StY species includes interspecific/generic introgressions and hybridization and chromosome structure rearrangements [Yang et al., 2016; Dou et al., 2017], and (3) with an unknown diploid donor of the $\mathrm{Y}$ genome and close genetic relationships with St, it is challenging to distinguish St and Y genomes based on genome in situ hybridization (GISH) [Liu et al., 2006; Yen and Yang, 2011]. Numerous reports demonstrated that various StY-containing species showed chromosome recombination; however, it is strenuous to identify interchromosomal (St-Y) and intrachromosomal (St-St, Y-Y) rearrangements [Dou et al., 2013; Yang et al., 2016; Liu et al., 2017; Wang et al., 2019]. Various cytogenetic and molecular methods were employed to identify the St and Y homoeologous and nonhomologous chromosomes. Random amplified polymorphic DNA (RAPD), sequencetagged site (STS), cleaved amplified polymorphism sequences (CAPS), and restriction fragment length polymorphism (RFLP) markers were applied to detect the St genome in controversial species and against common wheat genetic background [Zhang et al., 1998; Liu et al., 2006; Hu et al., 2015]. However, these markers failed to determine the genome constitution of species containing 2 or more St subgenomes. The recently described FISH probe $\mathrm{St}_{2}-80$ effectively identified the St genome in St- containing allopolyploids [Wang et al., 2017], but it was not sufficient in detecting nonhomologous chromosomes. Fewer Y genome probes were developed due to its unknown diploid donor. To determine the $\mathrm{Y}$ genome in polyploids, artificial hybridization with other Y-containing species can precisely identify the $\mathrm{Y}$ genome, but it is laborious and time-consuming. The STS marker successfully detected 1 Y nonhomologous chromosome against common wheat background, but it could not quantify the Y subgenome [Dou et al., 2012]. The St and Y genomespecific markers were developed and applied in wheat breeding programs [Zhang et al., 1998; Liu et al., 2006], but the lack of FISH probes limited the identification of individual St and Y nonhomologous chromosomes and chromosome structure variations. It is imperative to study novel StY-specific FISH markers that can identify St and Y nonhomologous chromosomes, elucidate the affinity between St and Y genomes, and demonstrate chromosome structure variations during polyploidization.

Roegneria grandis (Keng) is distributed in the mountain slopes of northwest China. The typical morphology of this species is erect spikes, and the spikelets are loosely arranged and cling to the cob. The palea is $1 / 3-1 / 4$ shorter than the lemma in length, the base part of the lemma is white and pilose, with hair up to $1 \mathrm{~mm}$. It is a tetraploid species, and the genome constitution is designed as $\mathrm{St}^{\mathrm{g}} \mathrm{S} \mathrm{g}^{\mathrm{g}} \mathrm{YY}$. Chromosome pairing behavior in meiosis $\mathrm{F}_{1}$ hybrids of $R$. grandis, Roegneria alashanica $\left(\mathrm{St}_{1} \mathrm{St}_{1} \mathrm{St}_{2} \mathrm{St}_{2}\right)$, and Elymus sibiricus (StStHH) indicated that the St genome of $R$. grandis, partly homologous with the St genome of $R$. alashanica and E. sibiricus, was modified and designed as $\mathrm{St}^{\mathrm{g}}$. Molecular phylogenetic analysis based on Accl gene further revealed that $\mathrm{St}^{\mathrm{g}}$ shared high similarity with the Y genome compared with other StY-containing species [Zhang et al., 2009]. The St and Y genomes are crucial in Triticeae, and tetraploid StY species participate extensively in polyploid speciation. However, the high genetic affinity between St and Y genomes and rare chromosome-specific markers inhibited the identification of nonhomologous chromosomes in StY-containing species.

The objective of our study was to identify St- and Y-enhanced repeat clusters, to understand the extent to which $S t^{g}$ differs from the St genome, whether it can be distinguished by the St genome markers. We also wanted to study whether homoeologous chromosomes between $\mathrm{St}^{\mathrm{g}}$ and $\mathrm{Y}$ can be accurately discerned due to high affinity. We employed comparative genome analyses to identify St- and Y-enhanced repeat clusters. Based on $\mathrm{St}_{2}-80$ and $45 \mathrm{~S}$ rDNA-specific markers and 4 newly developed repeat probes, we generated a FISH-based karyotype of $R$. grandis for the first time. 
Table 1. Species used in this study

\begin{tabular}{lllcc}
\hline Species & Accession & Origin of accession & $\begin{array}{l}\text { Chromosome } \\
\text { number }\end{array}$ & $\begin{array}{l}\text { Genome } \\
\text { symbol }\end{array}$ \\
\hline Pseudoroegneria spicata & PI537365 & United States National Plant Germplasm System & 28 & StSt \\
Pseudoroegneria stipifolia & PI531751 & United States National Plant Germplasm System & 28 & 28 \\
Roegneria ciliaris & GRA 1214 & Genbank of IPK & 28 & StSt \\
Roegneria grandis & ZY 3158 & Sichuan Agricultural University & StY \\
Triticum aestivum & - & Sichuan Agricultural University & ABD & A2 \\
\hline
\end{tabular}

\section{Materials and Methods}

\section{Materials}

Pseudoroegneria spicata PI537365 and Pse. stipifolia PI531751 were provided by the United States National Plant Germplasm System (Pullman, WA, USA). Roegneria ciliaris GRA 1214 was offered by the Genbank of the Leibniz Institute of Plant Genetics and Crop Plant Research (IPK, Gatersleben, Germany). R. grandis was collected by the researchers of the Triticeae Research Institute (Sichuan Agricultural University, Chengdu, China) (Table 1). The voucher specimens of all genotypes were deposited in the Herbarium of Triticeae Research Institute, Sichuan Agricultural University, China. Plants were grown under greenhouse conditions: $16 \mathrm{~h}$ light $/ 8 \mathrm{~h}$ dark and temperatures of $20-24^{\circ} \mathrm{C}$ day $/ 17-19^{\circ} \mathrm{C}$ night.

\section{Identification of StSt and StY Repeats Using Similarity-Based} Read Clustering

To estimate the genome size of Pse. stipifolia and R. ciliaris, flow cytometric genome size measurements were performed according to Ruban et al. [2020]. Genomic DNA of Pse. stipifolia (StStStSt) and $R$. ciliaris (StStYY) were isolated from leaves with DNeasy plant mini kit (Qiagen, Germany). Next-generation sequencing was conducted by Novogene (Beijing, China) (paired-end, $2 \times 101$ cycles, Illumina Hiseq-PE150 platform). In total, 1.4 and $1.5 \mathrm{~Gb}$ raw data were obtained from StSt and StY, respectively, corresponding to $0.2 \times$ genome coverage. In silico identification of St and $Y$ genome enriched candidate repeats was performed by similarity-based clustering of Illumina reads in the RepeatExplorer pipeline in the comparative mode (https://galaxy-elixir.cerit-sc.cz) [Novák et al., 2010, 2013]. Putative tandem repeats were compared with the database from National Center for Biotechnology Information (NCBI) by BLAST (program selection: megablast, https:// blast.ncbi.nlm.nih.gov/). Software Primer 7 was used for the design of primers (online suppl. Table 1; for all online suppl. material, see www.karger.com/doi/10.1159/000515623). The StSt/StY genomic DNAs were applied as templates, and PCR products were cloned into the PMD19-T vector according to the manufacturer's instructions (TaKaRa, China) and verified by sequencing (StY_34, StY_107, and StY_90). The PCR products of StY_34, StY_107, and StY_90wereprecipitatedby3 Msodiumacetate $\left(\mathrm{CH}_{3} \mathrm{COONa} \cdot 3 \mathrm{H}_{2} \mathrm{O}\right)$.

\section{Chromosome Preparation, Probe Labeling, and FISH}

Rapidly growing roots were collected from seedlings and adult plants. Roots were treated with nitrous oxide $\left(\mathrm{N}_{2} \mathrm{O}\right)$ at $0.1 \mathrm{MPa}$ for $2 \mathrm{~h}$ and then fixed with $90 \%(\mathrm{v} / \mathrm{v})$ glacial acetic for $5 \mathrm{~min}$. Afterward, roots were washed twice with distilled water. Meristems were digested with $4 \%$ cellulase and $2 \%$ pectolyasein in $0.01 \mathrm{M} \mathrm{ci-}$ trate buffer for $45 \mathrm{~min}$ at $37^{\circ} \mathrm{C}$. The preparation of mitotic chromosome spreads was performed according to Aliyeva-Schnorr et al. [2015] with minor modifications. Briefly, after enzymatic treatment, meristems were washed twice in $75 \%(\mathrm{v} / \mathrm{v})$ ethanol. Ethanol was replaced with $90 \%(\mathrm{v} / \mathrm{v})$ acetic acid $(10-15 \mu \mathrm{L}$ per root meristem), and meristems were disintegrated using a plastic pistil. The obtained suspension was dropped onto slides under $50-55 \%$ humidity conditions. After drying, slides were checked by phase-contrast microscopy and stored in $96 \%$ ethanol at $-20^{\circ} \mathrm{C}$ until used for FISH.

$\mathrm{St}_{2}-80$ [Wang et al., 2017] plasmid DNA and PCR products were labeled with dyes ATTO-488 or ATTO-550 by nick-translation using the ATTO NT Labeling Kit (Jena Bioscience, Germany). The StY_93- and pTa71-specific oligonucleotides were 5' labeled with 6-fluorescein amidite (FAM) or tetramethylrhodamine (TAMRA; Sangon Biotech, Shanghai, China) (online suppl. Table 2). Hybridization, detection, and visualization of FISH probes were conducted according to Wu et al. [2019]. Briefly, slides were UV crosslinked, $10 \mu \mathrm{L}$ of a hybridization cocktail $(1 \mu \mathrm{L}$ of labeled probe diluted in $9 \mu \mathrm{L}$ of $2 \times \mathrm{SSC} / 1 \times \mathrm{TE}$ buffer) was added per slide and covered with a coverslip. Specimens were denatured at $80^{\circ} \mathrm{C}$ for $5 \mathrm{~min}$ on a hot plate followed by incubation in a pre-warmed moist chamber at $55^{\circ} \mathrm{C}$ overnight. After coverslips were removed, the slides were washed in $2 \times$ SSC for 1 min. Finally, specimens were dehydrated and counterstained with 4',6-diamidino-2-phenylindole (DAPI) in Vectashield. For multiple rounds of FISH, coverslips were removed, and stripping was performed as follows: slides with specimen were boiled in $2 \times$ SSC for $10 \mathrm{~min}$, dehydrated for $10 \mathrm{~min}$ in each ethanol series, and air-dried. Those slides were exposed to intense sunlight for at least $24 \mathrm{~h}$. Hybridization signals were captured under the fluorescence Olympus BX61 microscope. All images were collected in greyscale and pseudo-colored with Adobe Photoshop CS (Adobe).

\section{Results}

\section{Genome and Karyotype Characteristics of Roegneria grandis (Keng)}

To detect the genome composition of allotetraploid $R$. grandis, FISH probe $\mathrm{St}_{2}-80$ was applied to distinguish the St genome. In R. grandis, 14 chromosomes displayed $\mathrm{St}_{2^{-}}$ 80 signals, which painted the entire chromosome except 


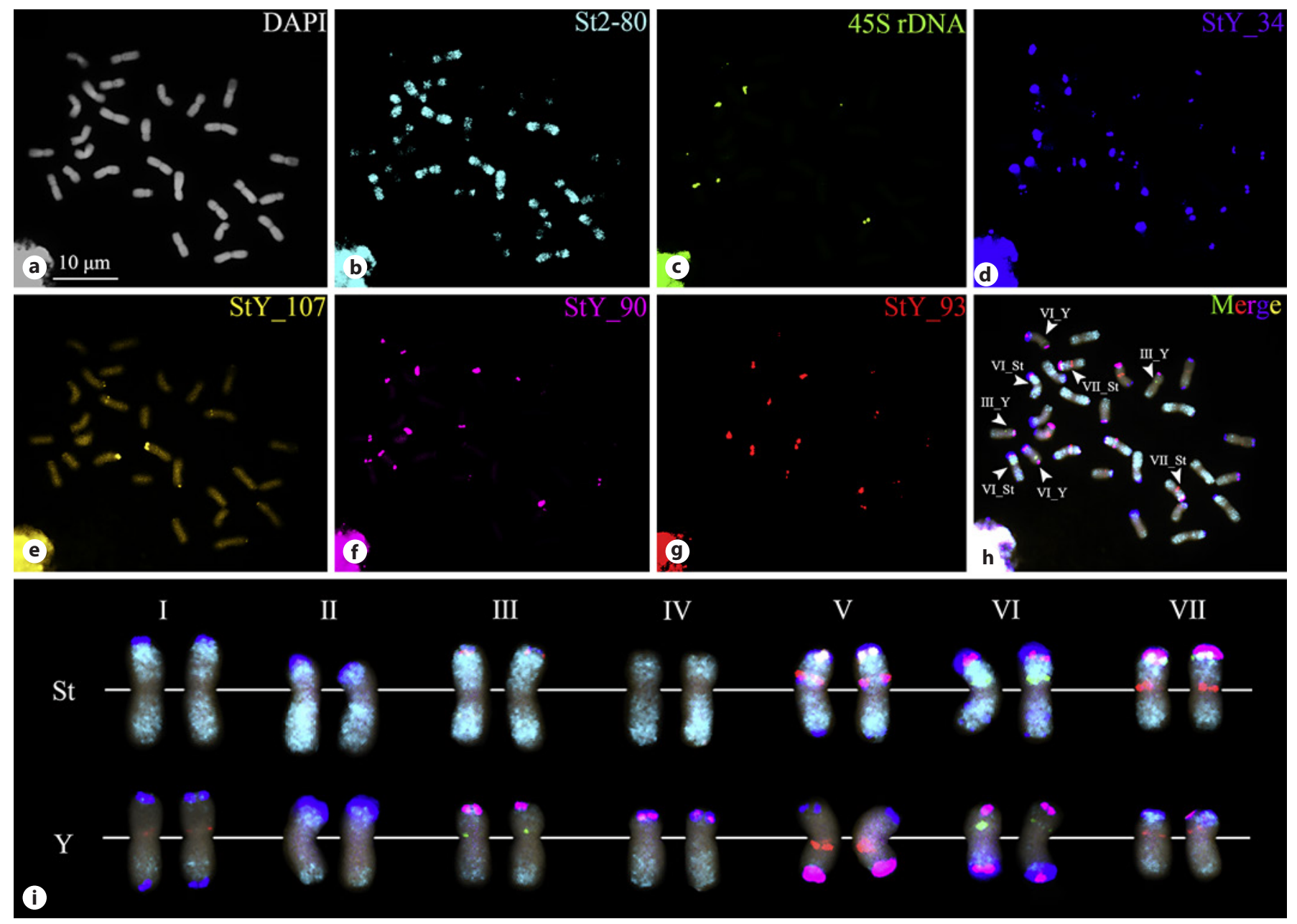

Fig. 1. FISH-based karyotype of Roegneria grandis. a DAPI staining. b-g Metaphase spread showing the specific FISH patterns of probes $\mathrm{St}_{2}-80$ (b), 45S rDNA (c), StY_34 (d), StY_107 (e), StY_90 (f), and StY_93 (g). h Merged signals of all probes. Chromosomes carrying 45S rDNA sites are marked with white arrowheads. i Karyotype of $R$. grandis arranged on the basis of chromosome length and distribution of probes. for the proximal region, while 14 chromosomes showed only fluorescence in the distal region (Fig. 1a, b). This result indicated that the genome symbol of $R$. grandis should be designed as StY [Zhang et al., 1998; Lei et al., 2016] rather than $\mathrm{St}^{\mathrm{g}} \mathrm{St}$ [Y $\mathrm{Yu}$ et al., 2010]. We used 15 well-spread mitotic metaphases to measure the entire length of the chromosome and each chromosome arm to establish the karyotype of $R$. grandis. Based on $\mathrm{St}_{2}-80$ signals, we divided the karyotype of $R$. grandis into St and Y subgenomes, and then ordered the nonhomologous chromosomes based on total chromosome length.

To locate the nucleolus organizer region (NOR) in $R$. grandis, we used the $45 \mathrm{~S}$ rDNA probe pTa71, generated from the wheat $45 \mathrm{~S}$ rDNA intergenic region. Four pairs of chromosomes showed pTa71 signal sites in R. grandis. Two of them were present in the St genome, and another 2 pairs were located in the Y genome (Fig. 1c, h, i). Based on the chromosome length, St chromosomes with the $45 \mathrm{~S}$ rDNA signal, scattered in the middle of the short arm and terminus of the short arm, were designed as VI_St and VII_St, respectively. In the Y genome, these were III_Y and VI_Y chromosomes, with $45 \mathrm{~S}$ rDNA located proxi$\mathrm{mal}$ and in the middle of the short arm, respectively. Our results showed the genome constitution of $R$. grandis as StStYY, and established the karyotype of this species based on subgenome and chromosome length. Interestingly, the number of $45 \mathrm{~S}$ rDNA sites differed from previous studies [Liu et al., 2017; Kong et al., 2018]. 

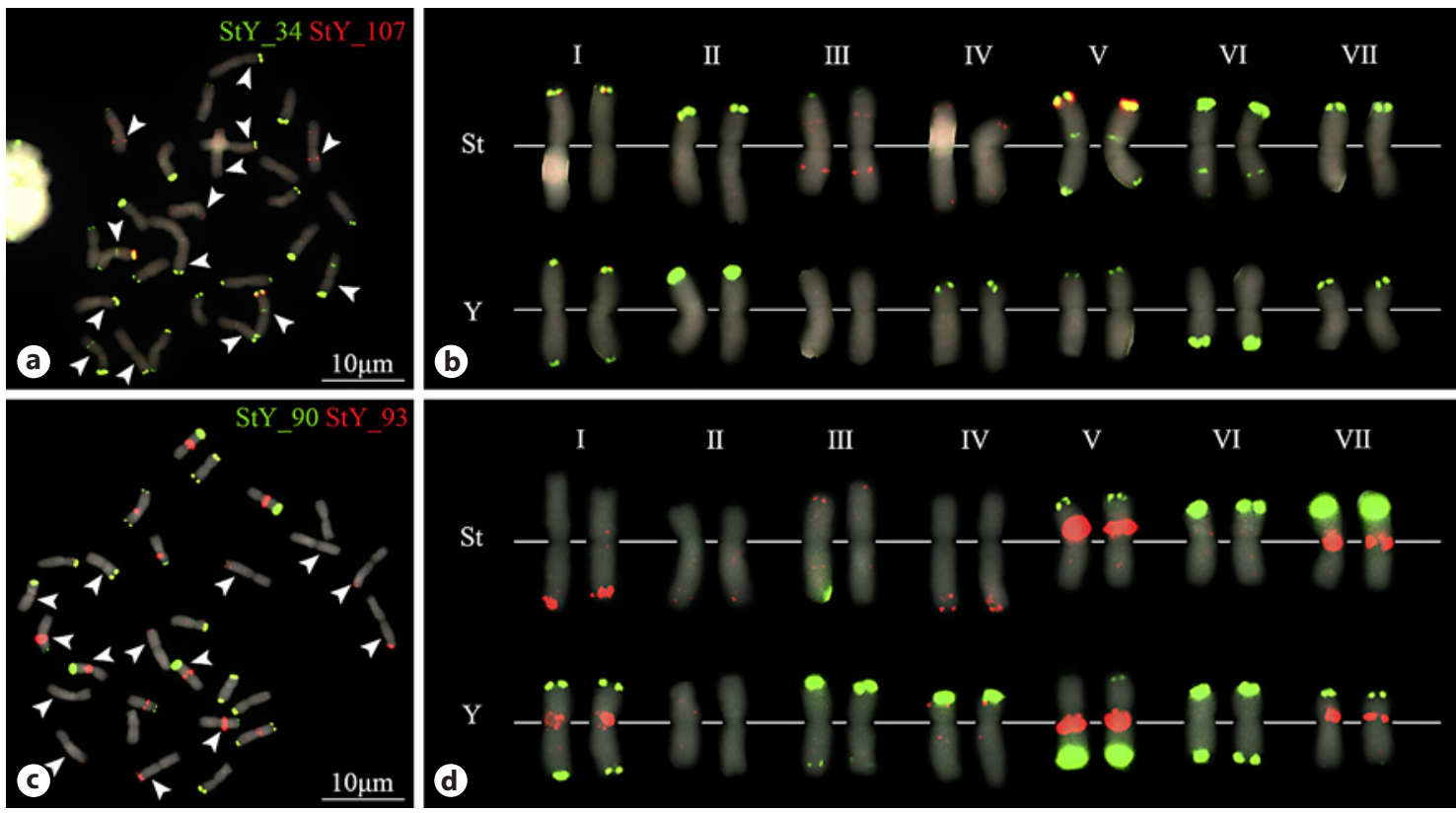

Fig. 2. Multi-color FISH on metaphase chromosomes of Roegneria grandis. a, c Mitotic metaphase spreads with StY_34 (green) and StY_107 (red) signals (a) and StY_90 (green) and StY_93 (red) signals (c). Chromosomes of the St genome are marked with white arrowheads. $\mathbf{b}, \mathbf{d}$ The chromosomes from $\mathbf{a}$ and $\mathbf{c}$, respectively, divided into St and Y subgenomes and arranged according to total chromosome length.

\section{Identification of St and Y Genome Repeat Clusters to}

Detect Specific Nonhomologous Chromosomes

To identify the repeat clusters beneficial to specific labeling of a complete set of St and Y nonhomologous chromosomes, we conducted comparative genomic analyses of StSt and StY, which were characterized by similaritybased clustering using the REPEATEXPLORER pipeline [Novák et al., 2013]. Four highly and moderately enriched random repeat clusters were found, which contained 2 St-enriched repeat clusters (named StY_34 and StY_107) and $2 \mathrm{Y}$-abundant repeat clusters (designed as StY_90 and StY_93) (online suppl. Fig. 1a-d ). Those newly putative satellites were aligned with the known repetitive sequences in the NCBI using BLASTN (https://blast.ncbi. nlm.nih.gov/Blast.cgi). To understand the characterization and distribution of the novel developed probes in $R$. grandis, we performed multiple rounds of double-colored FISH, which are described as follows:

\section{St-Enriched Repeat Sequences}

StY_34

Length $537 \mathrm{bp}, 0.450 \%$ genome proportion. It showed more than $90 \%$ similarity with the "Tail family" tandem repeat sequence in Triticeae species such as Leymus rac- emosus (Lam.) Tzvel., Secale cereale L., Agropyron cristatum (L.) Gaertn., Psathyrostachys juncea (Fischer) Nevski, Pse. stipifolia (Czern. Ex Nevski) Á. Löve, Aegilops tauschii Coss., and Triticum urartu Thum. Ex Gandil. The Tail family was first reported in the subtelomeric regions of $L$. racemosus [Kishii et al., 1999] and was later discovered in the centromeric region of common wheat [Kishii et al., 2001], thus showing a genus-dependent localization in one or the other region [Kishii and Tsujimoto, 2002]. In $R$. grandis, 24 chromosomes showed signals in the terminal, subterminal and/or proximal regions except for IV_St and III_Y chromosomes (Fig. 1d, h, i, 2a, b). In the St genome, the terminal regions of I_St_S (short arm), II_St_S, III_St_S, and VII_St_S showed signals; apart from both telomeric arm regions, V_St showed a signal in the pericentromeric region and VI_St displayed an intercalary signal in the long arm. In the Y genome, II_Y_S, IV_Y_S, V_Y_S, VI_Y_L (long arm), and VII_Y_S exhibited signals in their distal regions, and both terminal arms of I_Y showed signals. The V_St, VI_St, and I_Y chromosomes were distinguished based on the unique signal distribution pattern. We also detected the location of StY_34 in common wheat (Triticum aestivum c.v. Chinese Spring). It showed proximal signals in 24 
Fig. 3. The karyotype diagram of Roegneria grandis based on the distribution of $\mathrm{St}_{2}-80$ (light blue), 45S rDNA (green), StY_34 (blue), StY_107 (yellow), StY_90 (magenta), and StY_93 (red) repeats.

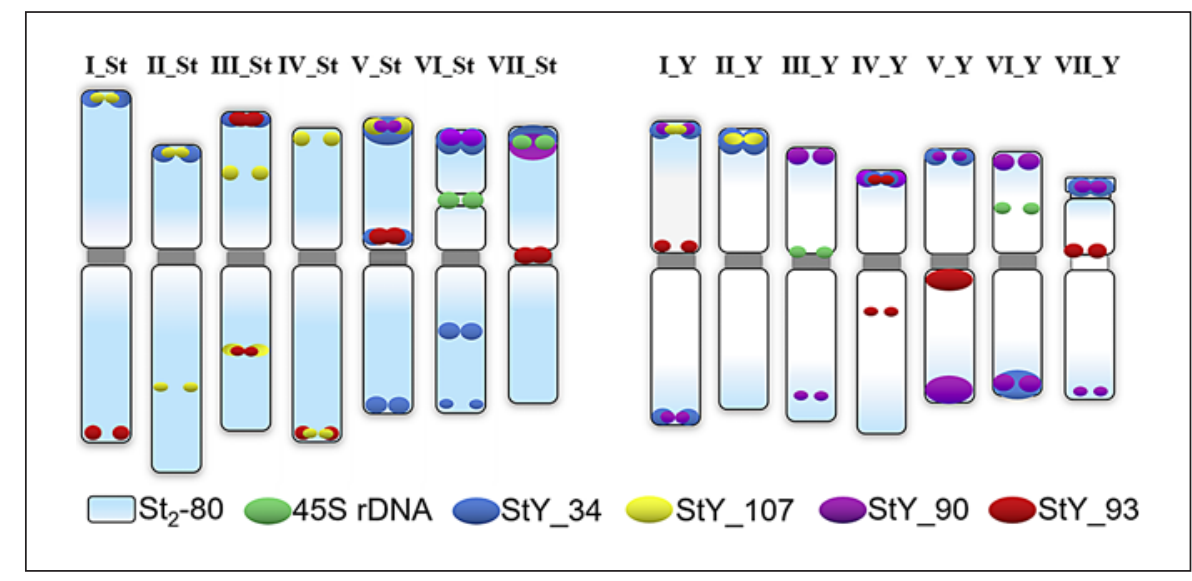

chromosomes of T. aestivum (online suppl. Fig. 2a-c), which differed from $R$. grandis where this probe was mainly located in the distal chromosome arm regions.

\section{StY 107}

Length $515 \mathrm{bp}, 0.100 \%$ genome proportion. It showed no sequence similarity with other species in the NCBI database. Fourteen chromosomes presented relatively less intense bands in the terminal or intercalary regions and unitary distribution signals than other repeats (Fig. 1e, h, i, 2a, b). The I_St_S, II_St, III_St, IV_St, and V_St_S in the St genome, and the distal region of I_Y_S and II_Y_S in the $\mathrm{Y}$ genome showed scattered StY_107 signals (Fig. 1e, h, i). The II_St, III_St, and IV_St exhibited unique band distribution and could be precisely detected. StY_107 showed no signal on T. aestivum, consistent with the NCBI blast result (online suppl. Fig. 2d-f).

\section{Y-Enriched Repeat Sequences}

\section{StY_90}

Length $348 \mathrm{bp}, 0.160 \%$ genome proportion. It shared $31 \%$ similarity with partial repeat sequence (DQ481558) of Lophopyrum elongatum (Host) Á. Löve when aligned in NCBI database. Eighteen chromosomes showed intense signals in the terminal chromosome regions (Fig. 1f, h, i, 2c, d). In the St genome, StY_90 was mainly distributed in the distal part of V_St_S, VI_St_S, and VII_St_S. In the Y genome, except chromosome II_Y, all other chromosomes presented StY_90 signals. V_St_S, VI_St, VII_St, I_Y, V_Y_L, VI_Y, and VII_Y_S were uniquely discovered by FISH signal intensity. Since, it showed no signal in common wheat, it served as a promising cytogenetic marker to identify the alien chromosomes against common wheat background (online suppl. Fig. $2 g-i$ ).

\section{StY_93}

Length $72 \mathrm{bp}, 0.140 \%$ genome proportion. It shared more than $92 \%$ sequence identity with 4 partial regions (AM932685, AM932684, AB924662, and FN564433) of common wheat $3 \mathrm{~B}$ chromosome and sequence of T. turgidum L. (EU835198). Twenty-two chromosomes exhibited intense signal variation in (peri)centromeric, proximal, subterminal, and distal regions (Fig. 1g, h, 2c, d). In R. grandis, StY_93 mainly showed intense signals in (peri) centromeric regions and scattered signals at the distal regions. StY_93 was distributed in the St genome on I_St_L, III_St, IV_St_L, proximal in V_St and VII_St. In the Y genome, StY_93 was located on the (peri)centromeres of I_Y, V_Y, and VII_Y, and both arms of IV_Y. In common wheat, StY_93 displayed intense signals on 28 chromosomes with an enhanced variation in the terminal, subterminal, intercalary, proximal, and (peri)centromeric regions (online suppl. Fig. 2j-1).

In summary, 4 repeat probes were scattered on individual St and Y chromosomes at variable locations that helped identify nonhomologous chromosomes of $R$. grandis (Fig. 1i). Moreover, StY_107 and StY_90 were promising probes for alien chromosome detection against the common wheat background.

Since multiple rounds of FISH might affect the hybridization efficiency of probes with less intensive signal, we conducted double-color FISH with StY_34 and StY_107, and StY_90 and StY_93 probes to confirm the signal location for these repeats on each chromosome (Fig. 2). Faint signals of StY_107 and StY_93 were visualized in doublecolor FISH. The combination of StY_34 and StY_107 labeled all St nonhomologous chromosomes; meanwhile, it also distinguished both arms of III_St, IV_St, V_St, and VI_St (Fig. 2a, b). StY_90 and StY_93 covered single 
chromosomes of the St and Y genomes except for II_Y. Although the signal location differed between St and Y genomes, it was also specific in the nonhomologous chromosomes; thus, the combination of StY_90 and StY_93 was applied to identify St and Y genomes and each nonhomologous chromosome (Fig. 2c, d). Our results helped to generate the idiogram karyotype of $R$. grandis (Fig. 3) and distinguished the complete set of StY nonhomologous chromosomes based on newly developed repeat clusters.

\section{Discussion}

Cytogenetic Karyotype of $\mathrm{R}$. grandis Based on Repeat Clusters

In our study, based on the St genome-enhanced probe $\mathrm{St}_{2}-80,45 \mathrm{~S}$ rDNA-specific markers, and 4 novel repeat probes (StY_34, StY_107,StY_90, and StY_93), we generated a FISH-based karyotype of $R$. grandis for the first time. These novel probes could specifically identify individual St and Y nonhomologous chromosomes. Löve [1984] suggested that species with the same genome or genome constitution should be classified into the same genus. The taxonomy of $R$. grandis was discussed controversially due to the genome constitution of this species designated as $\mathrm{StSt}^{\mathrm{g}}$ [Yu et al., 2010] or StgY [Zhang et al., 1998, 2009; Lei et al., 2016]. The recently developed FISH probe $\mathrm{St}_{2}-80$ made it possible to distinguish the St from the $\mathrm{Y}$ genome in StY-containing species [Wang et al., 2017]. Chromosome structure variations such as homoeologous recombination and gene conversion have been intensively investigated between different genomes in allopolyploids, resulting in difficulties in distinguishing between genomes with close genetic relationships such as St and Y [Liu et al., 2006]. However, the shortage of variable homoeologous and nonhomologous St and Y chromosome probes impeded the establishment of a FISH-based karyotype of $R$. grandis; moreover, it was insufficient to identify genome modification and chromosome structure variation.

In this study, 14 chromosomes showed intense $\mathrm{St}_{2}-80$ signals, which implied the StY genome constitution in $R$. grandis. Based on the comparative analysis model of the RepeatExplorer pipeline [Novák et al., 2013], we identified 4 graph-based tandem repeat clusters in StY species. StY_34 and StY_107 showed St genome enhancement, and StY_90 and StY_93 exhibited Y genome abundance. According to the signal distribution and intensity of $\mathrm{St}_{2}-$ $80,45 \mathrm{~S} \mathrm{rDNA}$, and 4 repeats, we constructed the first

Identification of Nonhomologous

Chromosomes
FISH-based karyotype of $R$. grandis. Besides, StY_34, StY_107, StY_90, and StY_93 determined each nonhomologous chromosome and both arms of each chromosome except VII_St_L and II_Y_L with scarce signal distribution. StY_107 and StY_90 exhibited signals in the common wheat, which indicated those probes could be used to identify alien chromosomes on the common wheat background (addition lines and substitution lines). The distribution and intensity of StY_34 and StY_93 varied within Triticeae species, which may illustrate that the rapid evolution of random repeats expressed speciespreference distribution and accumulation.

In StY species, the number of $45 \mathrm{~S}$ rDNA sites was relatively conserved in the St genome but variable in the $\mathrm{Y}$ genome. Liu et al. [2017] and Kong et al. [2018] uncovered 3 pairs of $45 \mathrm{~S}$ rDNA chromosomes in the StY species; 2 pairs were in the St genome, and 1 pair was in the Y genome. In this study, however, we identified 4 pairs of chromosomes, 2 in the St genome, and the others in the $\mathrm{Y}$ genome. The variant number of $45 \mathrm{~S} \mathrm{rDNA}$ sites in the genus Roegneria might result from polyphyletic speciation and geographic distribution [Liu et al., 2017]. The conserved rDNA sites such as $45 \mathrm{~S}$ rDNA are frequently detected on homoeologous group 1, 5, and 6 chromosomes of a few Triticeae species using FISH. In R. grandis, rDNA localizations were detected on chromosomes VI and VII in the St genome and chromosomes III and VI in the Y genome. This suggested that chromosomes VI and VII of the St genome might be homoeologous to the corresponding Y genome chromosomes, and they might belong to the homoeologous groups 1, 5, and 6 of common wheat, respectively. However, the lack of cytogenetic markers misled the identification and nomenclature of homoeologous chromosomes within Triticeae species. In $R$. ciliaris, Kong et al. [2018] identified 45S rDNA carrying chromosomes as first and 5St, and $5 \mathrm{Y}$ chromosome, while Liu et al. [2017] designed as first and 6St, and 7Y chromosomes. Thus, it is reasonable for species without a reference genome to establish a cytogenetic karyotype based on chromosome length. For more precise cytogenetic karyotype establishment, they should be based on the co-linearity of genes and should use well-established close relatives as a reference [Danilova et al., 2017; Parisod and Badaeva, 2020; Li et al., 2021].

\section{The Affinity between St and Y Genome}

Based on similar probe distribution within nonhomologous chromosomes (V_St, VII_St, I_Y, V_Y, VII_Y), we observed a high affinity between St and Y subgenomes; however, whether the close relationship arose from chro- 
mosome rearrangement during allopolyploidization needs further investigation. In $R$. grandis, the $\mathrm{St}^{\mathrm{g}}$ genome was a modified form of the St genome in Pseudoroegneria and showed homoeology with the Y genome in Roegneria [Zhang et al., 2009; Liu et al., 2017; Wang and Jensen, 2017]. For the relationship between St and Y genomes, there are 2 controversial opinions. Bivalent chromosome pairing, molecular markers, and gene sequences supported that the 2 distinct St and Y genomes originated independently from different donors [Dewey, 1980; Jensen, 1990; Torabinejad and Mueller, 1993; Gao et al., 2014; Hu et al., 2015]. Conversely, data of nuclear ribosomal internal transcribed spacer (nrITS) sequences, STS markers, and high-molecular-weight glutenin subunit (HMW-GS) gene data suggested that the $\mathrm{Y}$ genome shares a progenitor genome (designated $\mathrm{St}^{\mathrm{Y}}$ ) with the St genome of Pseudoroegneria with gradual differentiation [Liu et al., 2006; Okito et al., 2009; Zhang et al., 2016]. In this study, we found that StY_107 was enhanced in the St genome in silico and presented no similarity with other species in the NCBI database. However, it was detected in I_Y and II_Y chromosomes with a faint FISH signal. StY_93 was supposed to be a Y-enhanced repeat distributed in genome conserved (peri)centromere regions. It appeared in (peri) centromeric regions of V_St and VII_St, similar to I_Y, V_Y, and VII_Y. Thus, we presumed that St and Y were genetically related genomes. The high similarity of probe types and signal distribution patterns might be related to transposon insertion during polyploidization among $\mathrm{V}_{-}$ St and I_Y, VII_St and V_Y, and VII_Y. This is in accordance with previous researches in StY species based on $45 \mathrm{~S}$ rDNA FISH [Liu et al., 2017] and expressed sequence tag-simple sequence repeat markers [Kong et al., 2018], as well as in StYP species based on GISH and FISH [Dou et al., 2017]. In conclusion, clarifying the affinity between St and Y chromosomes will be conducive to chromosome structure rearrangement during allopolyploidization.

In this study, we explored 4 repeat clusters (StY_34, StY_107, StY_90, and StY_93) to identify St and Y chromosomes. However, rapid evolution causes the tandem repeats to diverge between species or populations, which does not facilitate comparative analyses between the closely related genome and genome combinations [Fu et al., 2015]. The gene order and locations on Triticeae chromosomes are relatively conserved, so it is reasonable to apply gene sequence-based FISH probes to identify the homoeologous chromosomes. Meanwhile, it facilitates the analysis of chromosome structure evolution in Triticeae [Danilova et al., 2014, 2017; Said et al., 2018; Naranjo, 2019; Parisod and Badaeva, 2020]. Our study lays foundation for (1) applying cDNA-FISH to $R$. grandis to uncover the precise karyotype based on colinearity of Triticeae species by using the common wheat karyotype as a reference, (2) analyzing diverse populations of the same species, to reveal intraspecific structural changes, and (3) generating the karyotype of different StY-containing species, to reveal the interspecific chromosome variation.

\section{Acknowledgment}

We thank the United States National Plant Germplasms System (Pullman, WA, USA) and the Genbank of the Leibniz Institute of Plant Genetics and Crop Plant Research (IPK) (Gatersleben, Germany) for providing seed material. We thank Dr. Joerg Fuchs (IPK, Gatersleben) for determining the genome size of $R$. ciliaris and Pse. stipifolia by flow cytometry, and Dr. Andreas Houben for insightful discussions.

\section{Statement of Ethics}

Ethical approval was not required for this study; no human or animal subjects were used.

\section{Conflict of Interest Statement}

The authors declare that they have no competing interests.

\section{Funding Sources}

This work was supported by the National Natural Science Foundation of China (NSFC, Grant Nos. 31470305 and 31870309).

\section{Author Contributions}

D.W. and Y.Z. conceived and designed the study. D.W., X.Z., and L.T. conducted experiments. D.W., X.Z., L.T., H.Z., and L.S. analyzed data. D.W., L.S., Y.W., H.K., X.F., J.L., and Y.Z. interpreted the data and wrote the manuscript. All authors read and approved the final manuscript.

References

Aliyeva-Schnorr L, Ma L, Houben A. A fast airdry dropping chromosome preparation method suitable for FISH in plants. J Vis Exp. 2015;106(106):e53470.

Danilova TV, Friebe B, Gill BS. Development of a wheat single gene FISH map for analyzing homoeologous relationship and chromosomal rearrangements within the Triticeae. Theor Appl Genet. 2014;127(3):715-30. 
Danilova TV, Akhunova AR, Akhunov ED, Friebe B, Gill BS. Major structural genomic alterations can be associated with hybrid speciation in Aegilops markgrafii (Triticeae). Plant J. 2017;92(2):317-30.

Dewey DR. Cytogenetics of Agropyron ugamicum and six of its interspecific hybrids. Int Plant Sci. 1980;141(3):305-12.

Dou QW, Lei Y, Li X, Mott IW, Wang RR. Characterization of alien chromosomes in backcross derivatives of Triticum aestivum $\times$ Elymus rectisetus hybrids by using molecular markers and sequential multicolor FISH/ GISH. Genome. 2012;55(5):337-47.

Dou Q, Wang RR, Lei Y, Yu F, Li Y, Wang H, et al. Genome analysis of seven species of Kengyilia (Triticeae: Poaceae) with FISH and GISH. Genome. 2013;56(11):641-9.

Dou Q, Yu F, Li Y, Zhao Y, Liu R. High molecular karyotype variation revealed in indigenous Elymus nutans in the Qinghai Plateau. Plant Divers. 2017;39(3):117-22.

Fu S, Chen L, Wang Y, Li M, Yang Z, Qiu L, et al. Oligonucleotide probes for ND-FISH analysis to identify rye and wheat chromosomes. Sci Rep. 2015;5(1):10552.

Gao G, Gou X, Wang Q, Zhang Y, Deng J, Ding $\mathrm{C}$, et al. Phylogenetic relationships and $\mathrm{Y}$ genome origin in Chinese Elymus (Triticeae: Poaceae) based on single copy gene DMC1. Biochem Syst Ecol. 2014;57:420-6.

Hu Q, Sun D, Sun G. Molecular phylogeny revealed distinct origin of the $\mathrm{Y}$ and St genome in Elymus longearistatus (Triticeae: Poaceae). Mol Phylogenet Evol. 2015;85:141-9.

Jensen KB. Cytology and taxonomy of Elymus kengii, E. grandiglumis, E. alatavicus, and E. batalinii (Poaceae: Triticeae). Genome. 1990; 33(5):668-673.

Kishii M, Tsujimoto H. Genus-specific localization of the Tail family of tandem-repetitive sequences in either the centromeric or subtelomeric regions in Triticeae species (Poaceae) and its evolution in wheat. Genome. 2002;45(5):946-55.

Kishii M, Nagaki K, Tsujimoto H, Sasakuma T. Exclusive localization of tandem repetitive sequences in subtelomeric heterochromatin regions of Leymus racemosus (Poaceae, Triticeae). Chromosome Res. 1999;7(7):519-29.

Kishii M, Nagaki K, Tsujimoto H. A tandem repetitive sequence located in the centromeric region of common wheat (Triticum aestivum) chromosomes. Chromosome Res. 2001; 9(5):417-28.

Kong L, Song X, Xiao J, Sun H, Dai K, Lan C, et al. Development and characterization of a complete set of Triticum aestivum-Roegneria ciliaris disomic addition lines. Theor Appl Genet. 2018;131(8):1793-806.
Lei Y-X, Zhang Y, Li Y-y, Fan X, Sha L-N, Wang $\mathrm{Y}$, et al. Phylogenetic analysis of the species with awnless lemma in Roegneria (Poaceae, Triticeae) based on single copy of nuclear gene DMC1. Biochem Syst Ecol. 2016;65: 185-91.

Li G, Zhang T, Yu Z, Wang H, Yang E, Yang Z. An efficient Oligo-FISH painting system for revealing chromosome rearrangements and polyploidization in Triticeae. Plant J. 2021; 105(4):978-93.

Liu QL, Ge S, Tang HB, Zhang XL, Zhu GF, Lu BR. Phylogenetic relationships in Elymus (Poaceae:Triticeae) based on the nuclear ribosomal internal transcribed spacer and chloroplast trnL-F sequences. New Phytol. 2006; 170(2):411-20.

Liu RJ, Wang RRC, Yu F, Lu XW, Dou QW. Characterization of genome in tetraploid $\mathrm{StY}$ secies of Elymus (Triticeae: Poaceae) using sequential FISH and GISH. Genome. 2017;60(8): 679-85.

Löve Á. Conspectus of the Triticeae. Feddes Repert. 1984;95(7-8):425-521.

Naranjo T. The effect of chromosome structure upon meiotic homologous and homoeologous recombinations in Triticeae. Agronomy. 2019;9(9):552-75.

Novák P, Neumann P, Macas J. Graph-based clustering and characterization of repetitive sequences in next-generation sequencing data. BMC Bioinformatics. 2010;11(1):378-90.

Novák P, Neumann P, Pech J, Steinhaisl J, Macas J. RepeatExplorer: a Galaxy-based web server for genome-wide characterization of eukaryotic repetitive elements from next-generation sequence reads. Bioinformatics. 2013;29(6): 792-3.

Okito P, Mott IW, Wu YJ, Wang RRC. A Y genome specific STS marker in Pseudoroegneria and Elymus species (Triticeae: Gramineae). Genome. 2009;52(4):391-400.

Parisod C, Badaeva ED. Chromosome restructuring among hybridizing wild wheats. New Phytol. 2020;226(5):1263-73.

Ruban A, Schmutzer T, Wu DD, Fuchs J, Boudichevskaia A, Rubtsova M, et al. Supernumerary $\mathrm{B}$ chromosomes of Aegilops speltoides undergo precise elimination in roots early in embryo development. Nat Commun. 2020; 11(1):2764-76.

Said M, Hřibová E, Danilova TV, Karafiátová M, Čížková J, Friebe B, et al. The Agropyron cristatum karyotype, chromosome structure and cross-genome homoeology as revealed by fluorescence in situ hybridization with tandem repeats and wheat single-gene probes. Theor Appl Genet. 2018;131(10):2213-27.
Torabinejad J, Mueller RJ. Genome constitution of the Australian hexaploid grass Elymus scabrus (Poaceae: Triticeae). Genome. 1993; 36(1):147-51.

Wang L, Shi QH, Su HD, Wang Y, Sha LN, Fan X, et al. St2-80: a new FISH marker for St genome and genome analysis in Triticeae. Genome. 2017;60(7):553-63.

Wang L, Jiang Y, Shi Q, Wang Y, Sha L, Fan X, et al. Genome constitution and evolution of Elytrigia lolioides inferred from Acc1, EF-G, ITS, TrnL-F sequences and GISH. BMC Plant Biol. 2019;19(1):158-72.

Wang RR, Jensen KB. Roegneria alashanica Keng: a species with the StStStYStY genome constitution. Genome. 2017;60(6):546-51.

Wang RR, Lu B. Biosystematics and evolutionary relationships of perennial Triticeae species revealed by genomic analyses. J Syst Evol. 2014; 52(6):697-705.

Wang RRC, Bothmer RV, Dvořák J, Fedak G Linde-Laursen I, Muramatsu M. Genome symbols in the Triticeae (Poaceae). In: Wang RRC, Jensen KB, Jaussi C, editors. Proceedings of the 2nd International Triticeae Symposium. Utah State University Publication Design and Production; 1994. p. 29-34.

Wu D, Ruban A, Fuchs J, Macas J, Novák P, Vaio $M$, et al. Nondisjunction and unequal spindle organization accompany the drive of Aegilops speltoides B chromosomes. New Phytol. 2019;223(3):1340-52.

Yang CR, Baum BR, Chen WH, Zhang HQ, Liu XY, Fan X, et al. Genomic constitution and taxonomy of the Chinese hexaploids Elymus cylindricus and E. breviaristatus (Poaceae: Triticeae). Bot J Linn Soc. 2016;182:650-7.

Yen C, Yang JL. Biosystematics of Triticeae. Beijing: Chinese Agriculture Press; 2011.

Yen C, Yang JL. Biosystematics of Triticeae. Beijing: Chinese Agriculture Press; 2013.

Yu HQ, Zhang C, Ding CB, Zhang HQ, Zhou YH. Genome constitutions of Roegneria alashanica, R. elytrigioides, R. magnicaespes and R. grandis (Poaceae: Triticeae) via genomic insitu hybridization. Nordic J Bot. 2010;28(2): 206-11.

Zhang C, Fan X, Yu H-Q, Zhang H-Q, Wang X-L, Zhou Y-H. Phylogenetic analysis of questionable tetraploid species in Roegneria and Pseudoroegneria (Poaceae: Triticeae) inferred from a gene encoding plastid acety1-CoA carboxylase. Biochem Syst Ecol. 2009;37(4):41220.

Zhang L, Li Z, Fan R, Wei B, Zhang X. Structural characterization and evolutionary relationship of high-molecular-weight glutenin subunit genes in Roegneria nakaii and Roegneria alashanica. Int J Mol Sci. 2016;17(7):1115-29.

Zhang XQ, Yang JL, Yen C. The genome constitution of Roegneria grandis (Poaceae, Triticeae). Plant Syst Evol. 1998;209:67-73. 